\title{
A Comparative Study of Peak Expiratory Flow Rate in Acute and Chronic Periodontitis
}

\author{
Thaslima Nandhini S. ${ }^{1}$, Gayatri Devi R. ${ }^{2}$ \\ ${ }^{1,2}$ Department of Physiology, Saveetha Dental College, Saveetha Institute of Medical and Technical Sciences \\ (SIMATS), Saveetha University, Poonamalle High Road, Chennai, Tamil Nadu, India.
}

\section{ABSTRACT}

\section{BACKGROUND}

Periodontal disease is a chronic inflammatory disease of gum which surrounds and supports the teeth. Globally, periodontal disease is one of the most common oral diseases. Unhealthy periodontium has been connected to systemic conditions like pulmonary and cardiovascular diseases. Periodontal disease affects individuals of all ages, but it is most commonly seen in elderly patients. According to the World Health Organization, nearly $65 \%$ of people have respiratory problems due to periodontal disease. Peak Expiratory Flow Rate (PEFR) is defined as the maximum rate and speed of expiration of an individual. We wanted to determine and compare PEFR in acute and chronic periodontitis.

\section{METHODS}

The present case control study was carried out among patients in the 20 - 40 years age group, who were attending outpatient department of Saveetha Dental College and Hospitals. Study participants were grouped into three categories as 'normal individuals', 'patients with acute periodontitis' and 'patients with chronic periodontitis'; each group had 20 people. Spirometer was used to detect the peak expiratory flow rate. Statistical analysis was done using SPSS. P value of less than or equal to 0.05 was taken as statistically significant.

\section{RESULTS}

Results were obtained and expressed as mean \pm SD. The Tukey HSD Post-hoc Test was used. Significance value is $<0.05$. PEFRs are $420 \pm 21.37$ and $317 \pm 21.05$ in control and periodontitis individuals, respectively. $\mathrm{P}$ values for these criteria were < 0.05. Males have a high PEFR when compared to females in normal individuals group. This difference was statistically not significant. Females have a high PEFR when compared to males in patients with acute periodontitis. This difference was statistically not significant. Males have a high PEFR when compared to females with chronic periodontitis. When compared within and between groups, it was statistically significant $(\mathrm{p}=0.0001)$.

\section{CONCLUSIONS}

Periodontitis could be a key source of respiratory disorders. This study shows that acute periodontitis expiratory flow rate was greater than chronic periodontitis expiratory flow rate. Proper maintenance of oral health and early detection of periodontitis may aid in reducing the frequency of respiratory problems due to periodontitis.

\section{KEY WORDS}

Acute Periodontitis, Chronic Periodontitis, Peak Flow Meter, Respiratory Disease

\author{
Corresponding Author: \\ Dr. Gayatri Devi R. \\ Department of Physiology, \\ Saveetha Dental College, \\ Saveetha Institute of Medical and Technical \\ Sciences (SIMATS), Saveetha University, \\ 162, Poonamalle High Road, \\ Chennai 600077, Tamil Nadu, India. \\ E-mail: gayatri.physio88@gmaiil.com
}

DOI: 10.14260/jemds/2020/724

How to Cite This Article:

Nandhini TS, Gayatri Devi GR. et al. A comparative study of peak expiratory flow rate in acute and chronic periodontitis. $J$ Evolution Med Dent Sci 2020;9(44):32943299, DOI: $10.14260 /$ jemds/2020/724

Submission 01-07-2020,

Peer Review 24-09-2020,

Acceptance 30-09-2020,

Published 02-11-2020.

Copyright (C) 2020 Thaslima Nandhini S. et al. This is an open access article distributed under Creative Commons Attribution License [Attribution 4.0 International (CC BY 4.0)] 


\section{BACKGROUND}

Periodontal disease is a chronic inflammatory disease of gum which surrounds and supports the teeth. Globally periodontal disease becomes one among the foremost common oral diseases. ${ }^{1}$ Unhealthy periodontium has been connected to systemic conditions like pulmonary diseases and cardiovascular disease. ${ }^{2}$ Due to poor oral hygiene, periodontitis is commonly seen among adults. Smoking is the major risk factors for periodontitis and COPD (Chronic Obstructive Pulmonary Disease). Oral pathogens like Porphyromonas gingivalis and Aggregatibacter take part a role in the respiratory diseases. In recent decades, unhealthy oral health, periodontal and respiratory illness association has been increasing. There is a less supporting study for a weak connection between chronic periodontitis and oral health. The relationship between respiratory health and periodontal health is not well stated, but the bacteria ${ }^{3}$ originating from the oral cavity are inhabited in the lungs. Mostly bacteria enter the lungs through microaspiration or spread of systemi c bacteria. Inflammatory mediators may travel from swollen gingiva to the lungs through blood. Hence, there is a biologically plausible reason for the connection between periodontal and lung health. ${ }^{4}$ The pathogens present in gingival sulcus and periodontal pockets in periodontal disease have direct entry into the systemic blood; moreover, they also shed in the saliva and are further carried down into the respiratory tract where they cause irritation of the respiratory mucosa increasing the risk of respiratory disease.

Periodontal disease affects people of all ages, but is most common in older patients. Severe infection of periodontal tissue can lead to infection of the tissues involved in periodontal disease like the gums, gingiva, periodontal ligament, cementum and alveolar bone. ${ }^{5,} 6$ Periodontitis is a common oral problem seen in patients visiting dentists. If a clear understanding of their relationship is understood, it will be easier for health care professionals to prescribe appropriate treatment as periodontitis is a preventable disease and can be avoided by awareness and modification of oral hygiene in patients. Therefore, a small amount of awareness can help to overcome the successive risk of respiratory illness. Although various studies have been reported in the literature on the same subject, the topic remains controversial.7, 8 The main area to be involved in this periodontal disease is gingival sulcus, a potential space found between the teeth and gums. ${ }^{9-11}$ According to the World Health Organisation, nearly $60 \%$ of people have respiratory illness due to periodontal disease. ${ }^{12}$ Increased bacterial load caused by poor oral hygiene and periodontal disease may also increase the risk of certain respiratory infections such as pneumonia and COPD. ${ }^{13}$ Pathogenic bacteria ${ }^{14,15}$ can colonize in the dental plaque biofilm that leads to respiratory disease. They can shed large amounts of secretions in the mouth and are in the form of cytokines and other life-sustaining molecules from the gingival gland. This secretion may aspirate into the lower respiratory tract and leads to disease and tenderness in the lungs. Patients with stress behaviour are at major and greater risk for severe periodontal disease.

Spirometry and peak expiratory flow rate measurements are the most widely used to assess airflow limitation. Although spirometry is an objective measure of airflow limitation, the peak flow meter (PFM) is a handy, easy-to-use, which can be an important aid in both monitoring and diagnosing of asthma. It measures the rate of pulmonary respiratory flow, which is the maximum airflow obtained during strong movement, with the lungs full of air. Peak expiratory flow rate is the most commonly used to monitor lung function. ${ }^{16,17}$ It is the main flow rate of expiration with a maximum effort from a peak endeavour of inspiration. It can be denoted as litres per min. ${ }^{18}$ PEFR depends on the effort and shows mainly bronchi and large bronchioles responsible for reflex bronchoconstriction. It is a good indicator of bronchial hyperactivity. ${ }^{19}$ At the age of 20 years, PEFR attains a peak, keeping the rate at about 60 years for women and decreasing with age. ${ }^{20}$ An important tool for determining the level of obstruction in the airways by measuring air flow through the bronchi. PEFR is influenced by a variety of factors such as age, gender and height. It can be used to confirm the diagnosis of respiratory disease (by measuring the reversibility bronchodilator and peak flow variability over a period of two weeks), to grade asthma severity, also for monitoring the response to pharmacotherapy. During respiratory disease there will be exacerbations, the PEF measurement serves as an important guide for disease management as spirometry may be contraindicated. Regular monitoring of pulmonary expiratory rates is especially useful among those who are 'poor perceivers' of their symptoms and among those who have severe respiratory symptoms like COPD and asthma. ${ }^{21}$ Early diagnosis by screening is crucial for preventive intervention and arresting respiratory disease progression during the management of a group of lung disease and have limited effectiveness. ${ }^{22}$

Wright peak flow meter was first introduced, and then later mini peak flow meter is commonly used to check the peak expiratory flow rate. Due to small and portable size, it can be handled easily by everyone without assistance. This is useful for detecting and monitoring the progression of pulmonary diseases. These measures the person's maximum breathing capacity called PEFR. If there is any obstruction and increased airway resistance in the respiratory tract, that could be reproduced in PEFR. ${ }^{23}$ Forced expiratory volume in one second is a forceful rate used in proper spirometry. ${ }^{24}$ It is a clear sign for hindrance in airway. Even though this flow rate usually positively associates with Forced expiratory volume 1 (FEV 1), this concentration decreases in patients with lung disease as airflow decreases. ${ }^{25}$ Peak flow rate can be used predominantly for the individuals elder than 5 years. It is most commonly measured with a convenient flow device and can be identified by a transducer that transfers the discharge flow during spirometry. Most of the previous studies were done on PEFR related to respiratory disease and very few numbers of studies were carried out other than respiratory problem. Over the past years various research done by our team are based on clinical reports, interventional studies, survey studies and systematic reviews. ${ }^{26-34}$ The purpose of this study is to evaluate the PEFR between acute and chronic periodontitis.

\section{METHODS}

\section{Study Participants}

The current case control study was carried out among 60 randomly selected patients in the age group of 20 - 40 years, who were attending outpatient departments of Saveetha 
Dental College and Hospitals during the period 2019 October December 2019. Sample size for this study depends on acceptable level of significance ( $p$ value), power $(1-\beta)$ of the study. The recommendation was submitted to the Saveetha Institute Ethics Committee and was approved. The Ethics Committee has approved the study on $20^{\text {th }}$ September 2019. Three groups of patients are selected with categories of normal individual, patients with acute periodontitis and patients with chronic periodontitis; every group has 20 individuals. Group I having normal healthy individuals, Group II having individuals with acute periodontal disease and Group III having individuals with chronic periodontal disease were included in this study (Group II and Group III patients are selected from the branch of Periodontics in Saveetha Dental College). For comparison and finding the differences between acute and chronic periodontitis, the estimated sample size of each group was 20 because this is pilot study.

\section{Inclusion Criteria and Exclusion Criteria}

The studies were evaluated and a complete standardized and systematic evaluation of all cases was performed. Appropriate history was taken like information on their families, socio economic conditions, habits, literacy and respiratory symptoms found and their quantity, the histories of their medicines have also been documented. Patients with acute and chronic periodontitis with the age between 20 - 40 years and both genders were involved in this study. Participants with the following diseases were rejected from this study which includes epilepsy, COPD, cardiac diseases, mentally retarded subjects and those who are not able to cooperative. Informal consents were obtained from all participants and ethical approval was obtained from the organisation. All the participants were non-smokers and non-athletes participating in related everyday activities. The ages of the subjects have been documented in years.

\section{Instrument}

Spirometry is used to detect the peak expiratory flow rate. The PEFR was recorded when the participants were settled comfortably. The commands and the procedures of the test were given / established to the patients before the study begins. Each patient underwent 3 times in PEFR manoeuvres and the utmost value was recorded, since this factor requires significant effort for recording. PEFR values were classified into three: Greater than $80 \%$ is the standard peak flow readings indicates that airway is healthy, normal peak flow readings between 50 to $79 \%$ indicates that there is less narrowing of airway and $<50 \%$ of the normal peak flow readings indicated modest or cruel narrowing of airway.

\section{Statistical Analysis}

All the data were expressed as mean \pm SE. The means were evaluated by one-way analysis of variance (ANOVA) with multiple comparison test of Tukey HSD Post-hoc test. Statistical analysis as well as plotting of graphs was carried out using SigmaPlot 13.0 (Systatsoftware, USA). P $<0.05$ was considered as significant.

\section{RESULTS}

Results were expressed as mean \pm SD. The Tukey HSD Posthoc test used; significance value is $<0.05$. By conventional criteria, this difference is considered to be not statistically significant. The age distribution of 20 - 40 years included in this study, the mean and standard deviation of their age (43.3 $\pm 9.24)$ were in normal individuals, $(43.3 \pm 8.14)$ in acute periodontitis and $(39.6 \pm 6.67)$ in chronic periodontitis. The mean and standard deviation of peak expiratory flow rate of normal individual ( $456 \pm 100.65)$, acute periodontitis (437 \pm 43.90) and chronic periodontitis ( $357 \pm 56.29$ ) (Table 1$)$. From figure 1 , the graph shows the mean of peak expiratory flow rate of a normal individual, normal individuals having higher PEFR than acute periodontitis. This difference was statistically not significant. The graph shows the mean of peak expiratory flow rate of chronic periodontitis, normal individuals having higher PEFR than chronic periodontitis. This difference was statistically significant. The graph shows the mean of peak expiratory flow rate of chronic periodontitis, acute periodontitis individuals having higher PEFR than chronic periodontitis. This variation was statistically insignificant. The difference was found not to be statistically significant when compared with normal and acute periodontitis. But when comparing with acute and chronic periodontitis, normal and chronic periodontitis were found to be extremely statistically significant. Group 1 vs. Group 2: Diff $=-19.0000,95 \% \mathrm{CI}=$ 73.2134 to 35.2134, p = 0.6778, Group 1 vs. Group 3: Diff = 99.0000, $95 \% \mathrm{CI}=-153.2134$ to $-44.7866, \mathrm{p}=0.0001$, Group 2 vs. Group 3: Diff $=-80.0000,95 \% \mathrm{CI}=-134.2134$ to 25.7866, $\mathrm{p}=0.0022$.

\begin{tabular}{|cccc|}
\hline & $\begin{array}{c}\text { Normal } \\
\text { (Group 1) }\end{array}$ & $\begin{array}{c}\text { Acute } \\
\text { (Group 2) }\end{array}$ & $\begin{array}{c}\text { Chronic } \\
\text { (Group 3) }\end{array}$ \\
Mean & 456 & 437 & 357 \\
Standard Deviation & 100.65 & 43.90 & 56.29 \\
\hline \multicolumn{4}{r}{ Table 1. Mean PEFR in Acute and Chronic Periodontitis } \\
\hline
\end{tabular}

\begin{tabular}{|ccc|}
\hline Groups & P Value & Significance \\
Group 1 vs. Group 2 & 0.6778 & Not Significant \\
Group 1 vs. Group 3 & 0.0022 & Significant \\
Group 2 vs. Group 3 & 0.0022 & Significant \\
\hline Table 2. Multiple Comparison by Tukey & HSD Post-Hoc Test \\
\hline
\end{tabular}

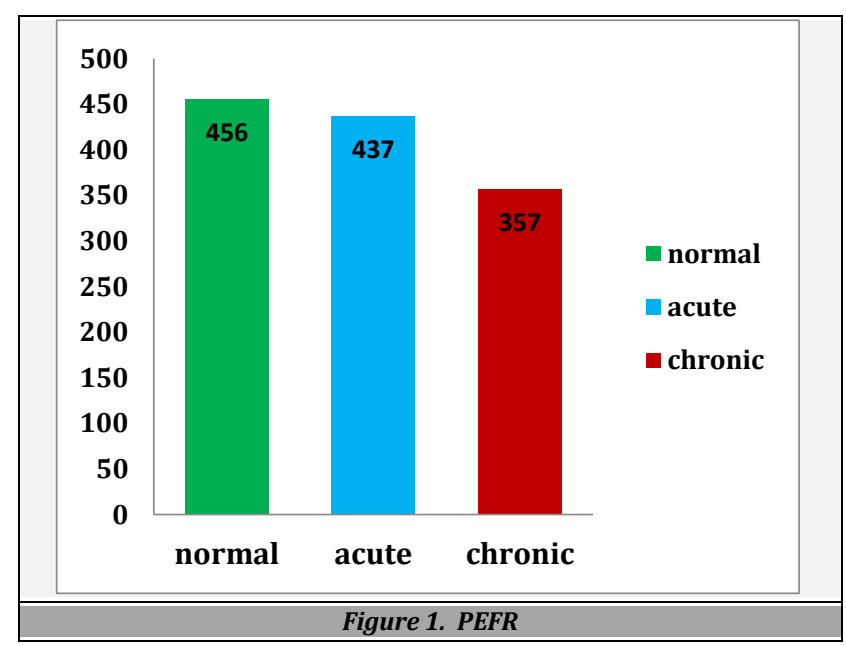

Figure 1. Mean of peak expiratory flow rate in normal individuals, acute and chronic periodontitis. Normal individuals having higher PEFR than acute periodontitis. $\mathrm{P}$ value for this Criterion was $p<0.05$. The difference between 
normal and acute was statistically not significant. The differences between normal and acute, acute and chronic periodontitis individuals were statistically significant.

\section{DISCUSSION}

The peak expiratory flow was determined among the acute and chronic periodontitis patients. The dispute is to distinguish high respiratory flow differences between acute and chronic periodontitis. Standard statistical tests have been found to be insensitive and non-specific due to variable periods for deterioration to occur after exposure and sometimes prolonged time for recovery to occur, in such days away from work initially have lower measurements.

We found that gender and age to be the largest determinants of variability in peak expiratory flow rate, which was consistent with the previous observations.35,36,37 We also found that females have lower PEFR values than males. We did not study the effect of weight, because its role in determining PEFR has been debated, because of two opposing effects like increased weight due to muscular development increases PEFR and increased weight due to obesity decreases PEFR. Periodontal poor health is often reported in patients with rhinitis and it is thought that this may be due to xerostomia occurring in the blocked nose, which may be a good source of bacteria that cause periodontitis. Some bacteria and microorganisms are transmitted from the oral cavity to the lungs, and thus the bacteria that live in the oral cavity, for example those related to periodontal disease, can affect respiratory life directly or indirectly through inflammation of the system, or carry common epithelial substances to both periodontal and respiratory. ${ }^{13}$ There are many reason linking the respiratory disease and periodontal problem which include bacterial spread, low immunity, others factors and inflammation. Oral bacteria which cause periodontal disease easily enter into lower respiratory tract. Once the bacteria reach the lungs, it can increase the growth thereby it cause pneumonia and other serious respiratory disorders like chronic obstructive pulmonary disease. For low immunity people, oral bacteria easily affects, colonize above and below the gum. It can easily accelerate the periodontal disease as well as increased the risk of emphysema, COPD and pneumonia. Smoking and tobacco users have higher risk of developing respiratory as well as periodontal problems. Oral bacteria cause inflammation of pleura, thereby it can restrict the airflow in and out of the lungs. Oral bacteria in secretions can bind to the respiratory epithelial mucosal surface. These oral bacteria which bound may prompt mucosal epithelium to secrete cytokines. Cytokines are attached to saliva, which can infect respiratory epithelial cells. Cells are stimulated and release other cytokines including inflammatory cells at the site. These provocative cells can release hydrolytic enzymes that can be at high risk of colon damage by respiratory bacteria.

Males have high PEFR when compared to women $630 \pm$ 15.64 and $600 \pm 21.11$, respectively. This variation in sexes is due to large thoracic cage, lung volume, and muscle strength. In periodontitis individuals, male had higher PEFR than females. From this study, it observed that normal individuals have higher PEFR than acute periodontitis individuals in both sexes. This may be due to airway contamination by inhaling the harmful particles or bacteria during breathing. Oral flora can be aspirated along with oral secretions, this may alter the cytokine composition, thereby it affects the respiratory mucosa or epithelium. ${ }^{38}$ There are numerous mechanisms studied to explain the function of oral bacteria in respiratory infection pathogenesis. While breathing, pathogens in the oral cavity enter into the lung thereby it leads infection; Some associated enzymes with periodontal disease in saliva may change the surface of mucosa, in turn it boost respiratory pathogens adhesion and colonization, then pathogens enter into the lung; enzymes linked to periodontal disease may destroy salivary protein layer to block their clearance from the surface of mucosal; and cytokines from periodontal tissues may modify the respiratory epithelium, thereby promoting respiratory pathogens. The correlation between periodontal disease and chronic obstructive lung disease has been found to be the fourth leading cause of mortality in the United States. These findings accentuate the necessity for developing oral hygiene who are at risk and those living in long-term care institutions. Oral diseases especially periodontitis should be considered on preference basis to protect the overall health and thereby it curtail the risk for systemic infections and diseases.

We conclude that peak expiratory flow rate, which is considered even in asymptomatic states as an early determination of airway obstruction, is a very simple procedure that does not require any sophisticated, expensive instrument or assistance; it could be routinely performed and may be done twice a year. The limitation of our research is its limited sample size, we can suggest that this analysis should be carried out on a larger sample scale to increase the study's efficiency. Although proper instructions have been given and all recordings have been done by the same person during the research, the chances of obtaining false positive results cannot be denied as the PEFR depends on effort. Studies which correlate PEFR with different parameters of spirometry can be planned to identify false positive cases. We may also compare PEFR values with another lung function test which is simple to perform.

\section{CONCLUSIONS}

Periodontitis is a key source of respiratory illness. Periodontal disease is one of the most common oral diseases in the world. Periodontal poor health has been linked to systemic conditions such as diabetes, pulmonary disease and heart disease. The study aims to compare PEFR with acute and chronic periodontitis and the results conclude that PEFR rates were lower in patients with chronic periodontitis compared to patients with acute periodontitis. This indicates that chronic periodontitis is an important risk factor for airflow limitation and lung function in periodontal compromised patients. Hence regular monitoring of PEFR, should be done in periodontitis patients as it is an important tool to assess the effect of disease. This study showed that chronic periodontitis has a lower expiratory flow rate than acute periodontitis. Therefore, it would be beneficial to reduce the rate of occurrence of respiratory diseases related to periodontitis by early detection of periodontitis, as well as by maintaining good oral health and hygiene. 
Data sharing statement provided by the authors is available with the full text of this article at jemds.com.

Financial or other competing interests: None.

Disclosure forms provided by the authors are available with the full text of this article at jemds.com.

\section{REFERENCES}

[1] Rajesh H, Boloor V, Rao A, et al. Knowledge of periodontal disease among group of health care professionals in Yenepoya University, Mangalore. J Educ Ethics Dentistry 2013;3(2):60-5.

[2] Oberoi SS, Harish Y, Hiremath S, et al. A cross- sectional survey to study the relationship of periodontal disease with cardiovascular disease, respiratory disease and diabetes mellitus. J Indian Soc Periodontol 2016;20(4):446-52.

[3] Dickson RP, Erb-Downward JR, Freeman CM, et al. Bacterial topography of the healthy human lower respiratory tract. American Society for Microbiology 2017;8(1):e02287-16.

[4] Real FG, Barrionuevo LP, Franklin K, et al. The association of gum bleeding with respiratory health in a population based study from Northern Europe. PLOS One 2016;11(1):e0147518.

[5] Jordan RCK. Diagnosis of periodontal manifestations of systemic diseases. Periodontol 2000;34:217-29.

[6] Albandar JM, Rams TE. Global epidemiology of periodontal diseases: an overview. Periodontol 2000;29:7-10.

[7] Dongxue Z, Zuomin W. The Association between chronic periodontitis and chronic obstructive pulmonary disease - a review. Int J Oral Dent Health 2018;4(2):062.

[8] Gomes-Filho IS, Passos JS, da Cruz SS. Respiratory disease and the role of oral bacteria. J Oral Microbiol 2010;2(1):5811.

[9] van Palenstein Helderman WH, Joarder MA, Begum A. Prevalence and severity of periodontal diseases and dental caries in Bangladesh. Int Dent J 1996;46(2):76-81.

[10] Ronderos M, Pihlstrom BL, Hodges JS. Periodontal disease among indigenous people in the Amazon rain forest. J Clin Periodontol 2001;28(11):995-1003.

[11] Albandar JM, Brunelle JA, Kingman A. Destructive periodontal disease in adults 30 years of age and older in the United States, 1988-1994. J Periodontol 1999;70(1):13-29.

[12] Moghadam SA, Shirzaiy M, Risbaf S. The associations between periodontitis and respiratory disease. J Nepal Health Res Counc 2017;15(1):1-6.

[13] Armitage GC. Periodontal diagnoses and classification of periodontal diseases. Periodontol 2000;34:9-21.

[14] Moore WE, Moore LV. The bacteria of periodontal diseases. Periodontol 2000;5:66-77.

[15] Agarwal S, Gangaram D, Saleem M. Periodontitis in patients with type 2 diabetes mellitus. Ann Inter Med Dent Res 2018;4(3):58-63.

[16] Kulie T, Slattengren A, Redmer J, et al. Obesity and women's health: an evidence-based review. J Am Board Family Med 2011;24(1):75-85.

[17] Mafort TT, Rufino R, Costa CH, et al. Obesity: systemic and pulmonary complications, biochemical abnormalities and impairment of lung function. Multidiscip Respir Med 2016;11:28.

[18] Standardization of Spirometry, 1994 update. American Thoracic Society. Am J Respir Critical Care Med 1995;152(3):1107-36.

[19] Shenoy J, Shivakumar J, Bhat S, et al. Impact of adiposity markers on peak expiratory flow rate in young adult South Indian females. Muller J Med Sci Res 2014;5(2):121-4.

[20] Mishra DJ, Mishra J. Variations in PEFR among males and females with respect to anthropometric parameters. IOSR J Dent Med Sci 2013;5(1):47-50.

[21] Global Strategy for asthma management and prevention. NHLBI/WHO Workshop Report 1995: p. 176.

[22] Chakrabarti P. Early, Easy, inexpensive diagnosis an urgent need for global TB control. 2007 Frontiers in the Convergence of Bioscience and Information Technologies 2007. http://dx.doi.org/10.1109/fbit.2007.153

[23] Bumatay A, Chan R, Lauher K, et al. Coupled mobile phone platform with peak flow meter enables real-time lung function assessment. IEEE Sensors J 2012;12(3):685-91.

[24] Thiadens HA, De Bock GH, Van Houwelingen JC, et al. Can peak expiratory flow measurements reliably identify the presence of airway obstruction and bronchodilator response as assessed by $\mathrm{FEV}(1)$ in primary care patients presenting with a persistent cough? Thorax 1999;54(12):1055-60.

[25] Gibson PG. Monitoring the patient with asthma: an evidence - based approach. J Allergy Clin Immunol 2000;106(1 Pt 1):17-26.

[26] Samuel AR, Devi MG. Geographical distribution and occurrence of endemic goitre. Res J Pharm Tech 2015;8(8):973-8.

[27] Baheerati MM, Gayatri Devi R. Obesity in relation to infertility. Research J Pharm Tech 2018;11(7):3183-5.

[28] Harsha L, Priya J, Shah KK, et al. Systemic Approach to management of neonatal jaundice and prevention of kernicterus. Res J Pharmacy Tech 2015;8(8):1087-92.

[29] Abigail, Jyothi P, Devi G. Evaluation of muscular endurance among dentists. Indian J Public Health Res Develop 2019;10(10):258-61.

[30] Priya AJ, Devi G. Physical fitness among the dental physician, dental undergraduates and postgraduates students. Indian Journal of Public Health Research \& Development 2019;10(10):223-6.

[31] R GD, Sethu G. Evaluation of adenoids by oronasal and nasal spirometry. Asian J Pharmaceutical Clin Res 2018;11(10):272.

[32] Timothy CN, Devi RG, Priya AJ. Evaluation of peak expiratory flow rate (PEFR) in pet owners. Indian J Public Health Res Develop 2019;10(8):803.

[33] Rj I, R GD. Role of environmental factors on sleep patterns of different age groups. Asian J Pharmaceutical Clin Res 2016;9(6):124.

[34] Iyer PK, Devi RG, Priya AJ. A survey study on causes, treatment and prevention of onychocryptosis. Indian Public Health Res Develop 2019;10(8):807.

[35] VijayanVK, Kuppurao KV, Venkatesan P, et al. Pulmonary function in healthy young adult Indians in Madras. Thorax 1990;45(8):611-5.

[36] Mondal H, Mondal S, Panigrahi AK, et al. Applicability of established regression equations in the prediction of peak 
expiratory flow rate in Indian adults. Indian J Allergy Asthma Immunol 2019;33(1):39-44.

[37] Ray D, Rajaratnam A, Richard J. Peak expiratory flow in rural residents of Tamil Nadu, India. Thorax 1993;48(2):163-6.
[38] Indian Genome Variation Consortium. Genetic landscape of the people of India: a canvas for disease gene exploration. J Genet 2008;87(1):3-20. 